\title{
Images of Turbulence in a Tokamak Plasma
}

\author{
G. R. McKee, C. Fenzi, and R. J. Fonck
}

\begin{abstract}
Two-dimensional measurements of density fluctuations in the DIII-D tokamak, obtained with the Beam Emission Spectroscopy diagnostic, have allowed for construction of images and movies of plasma turbulence which are greatly aiding an understanding of tokamak turbulence phenomena. The measurements are obtained in the radial and poloidal plane at a spatial resolution of near $1 \mathrm{~cm}$ (about five ion gyroradii). Assembly of synchronized discrete measurements provides a low spatial resolution, high sensitivity, high throughput, high time resolution $(\Delta t=1 \mu \mathrm{s})$ imaging system. These measurements have shed light on such processes as flow shear, turbulent eddy interaction and shearing, and provide direct visualization of long wavelength turbulence in a hot plasma.
\end{abstract}

Index Terms-Density fluctuations, image analysis, plasma measurement, plasma turbulence, tokamak.

$\mathbf{T}$ HE LARGE pressure gradients in magnetically confined fusion-grade plasmas result in significant plasma turbulence. This turbulence is essentially a two-dimensional (2-D) phenomenon in tokamaks, and so its full characterization requires measurement of fluctuations in these relevant dimensions. Plasma turbulence in tokamaks is of great scientific interest because of the large anomalous cross-field transport of energy, momentum, and particles that results from correlated density, temperature, and electrostatic and/or magnetic fluctuations [1]. Turbulence images are desired for a number of scientific objectives: visualization of turbulent structures, measurement of shear flows and the turbulent velocity field, and ultimately to quantify the nonlinear transfer of energy within the turbulent spectrum [2]. The turbulence is considered 2-D because the correlation lengths in the radial and poloidal directions are typically short, of order five to ten ion gyroradii $(\sim 2 \mathrm{~cm})$, while the toroidal correlation length is typically comparable to the device size $(\sim 1 \mathrm{~m})$ and so can be ignored.

To address these issues, the Beam Emission Spectroscopy diagnostic system [3], which measures spatially localized density fluctuations, has been deployed on the DIII-D tokamak and configured to provide 2-D measurements in the relevant radial and poloidal directions [4]. Density fluctuations are observed by measuring the collisionally-induced emission of the Doppler-shifted $n=3-2$ transition (Balmer series $D_{\alpha}$ at $\lambda_{o}=656.1 \mathrm{~nm}$ ) of the high-energy deuterium neutral beams that are injected to heat, fuel, and rotate tokamak plasmas.

Manuscript received June 29, 2001; revised November 15, 2001.

G. R. McKee is with the University of Wisconsin-Madison, Madison, WI 53706 USA. He is also with General Atomics, Office 13-367, San Diego, CA 92186-5608 USA(e-mail: mckee@fusion.gat.com).

C. Fenzi was with the University of Wisconsin-Madison, Madison, WI 53706.

She is now with CEA, Association Euratom-CEA, Cadarache 13108, France.

R. J. Fonck is with the University of Wisconsin-Madison, Madison, WI 53706 USA.

Publisher Item Identifier S 0093-3813(02)03074-6.
The neutral beam and optical viewing geometry provide measurements at a spatial resolution of $\Delta r=\Delta z \approx 1 \mathrm{~cm}$ with roughly a $1-\mathrm{cm}$ channel-to-channel separation. Thirty-two spatial channels are deployed allowing for 2-D measurements on a $5-\mathrm{cm} \times 6-\mathrm{cm}$ grid that can be radially scanned. Measurements are sampled at $1 \mathrm{MHz}$ and the broadband density fluctuations typically exist up to $\sim 250 \mathrm{kHz}$ in the outer regions of the plasma discussed here. Each channel has very high throughput and high efficiency, as required to measure these relatively small fluctuation signals, and provides a $0.5 \mathrm{~s}$ time record. Thus, such measurements cannot be readily achieved with higher resolution, lower throughput, high-speed cameras. Two-dimensional turbulence images have also been obtained in relatively cold plasmas using Langmuir probe arrays [5].

A temporal sequence of images of plasma turbulence obtained in a low-confinement mode plasma are shown in Fig. 1. Each frame, integrated for $\Delta t=1 \mu \mathrm{s}$, is separated in time by $10 \mu \mathrm{s}$. The measurements were obtained in the spatial region $\rho=0.9-1.05$ at the outer midplane ( $\rho$ is the normalized toroidal magnetic flux radial coordinate). The images are constructed from the raw data as follows. The turbulence exists over the frequency range $f<250 \mathrm{kHz}$. This data was, therefore, frequency-filtered over $1-250 \mathrm{kHz}$ (maintaining constant phase) to minimize extraneous electronic and photon noise in the signal at higher frequencies, as well as to avoid low-frequency beam noise $(<1 \mathrm{kHz})$. The $5 \times 6$ grid of data is then interpolated to a $50 \times 60$ grid using a minimum curvature surface 2-D spline method, and finally the data is linearly interpolated to a $250 \times 300$ pixel image for display. The color scheme is such that green represents the equilibrium density and red and blue represent positive and negative fluctuations about the equilibrium. The RMS magnitude of density fluctuations, $\tilde{n} / n$, range from $4 \%$ to $15 \%$ over the radial region sampled, with densities in the range $n \approx 1.5-2.5 \times 10^{19} \mathrm{~m}^{-3}$, ion temperatures near $200 \mathrm{eV}$, and electron temperatures near $100 \mathrm{eV}$.

Movies are generated from the 2-D data by assembling a temporal sequence of images, each separated by $1 \mu \mathrm{s}$. Radial and poloidal correlation lengths of the turbulenceare in the range of $1-3 \mathrm{~cm}$, and decorrelation times are of order $10 \mu \mathrm{s}$. The time-averaged eddy motion is in the poloidal (vertical) direction as a result of equilibrium $E_{r} x B_{T}$ and diamagnetic flows [indicated by white arrows in Fig. 1(a)], with essentially no net steady-state radial motion. Typical poloidal velocities are of order $5-10 \mathrm{~km} / \mathrm{s}$ and have a significant radial gradient. These velocities are measured using time-delay correlation analysis between poloidally separated spatial channels. A radial shear in the poloidal flow velocity is typically evident in this edge region of the plasma. Such flow shears can attain values of $d v_{\theta} / d r \approx 5 \mathrm{~km} / \mathrm{s} / \mathrm{cm}$ or $5 \times 10^{5} \mathrm{~s}^{-1}$, comparable to the measured nonlinear decorrelation rates of the turbulence. 


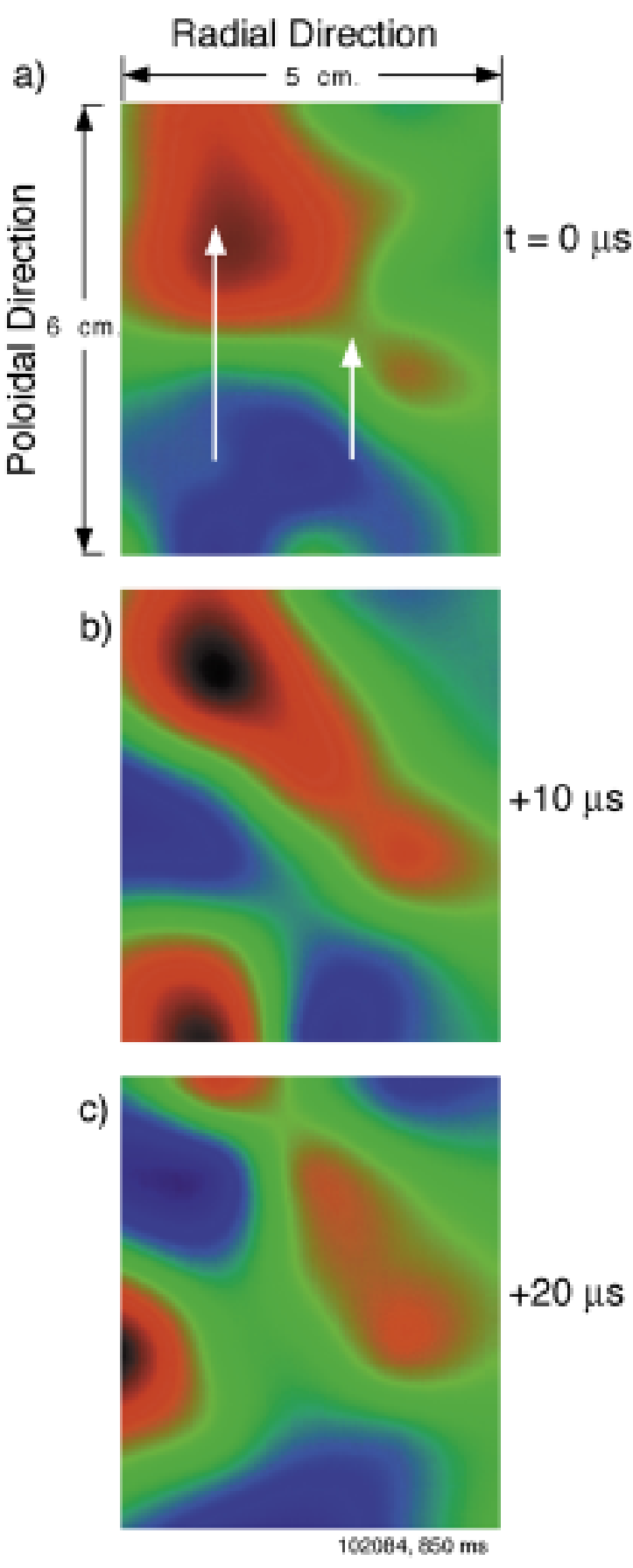

Fig. 1. Images of density fluctuations at $10-\mu$ s intervals, obtained over a $5-\mathrm{cm}$ (radial) $\times 6-\mathrm{cm}$ (poloidal) region at the outer plasma midplane, $\rho=0.9-1.05$, in a low-confinement ( $L$-mode) discharge. White arrows in (a) qualitatively indicate average flow direction and magnitude.

Eddy shapes evolve in intricate patterns and interact nonlinearly with neighboring eddies. Large eddies often are torn apparent as a result of the poloidal flow shear, while smaller eddies often join together. In Fig. 1(a), the large "red" eddy in the upper left initially has a roughly round shape, but $10 \mu$ s later, in
Fig. 1(b), the turbulent structure has evolved into a more diagonally elongated blob in response to the radial shear of poloidal flow. By Fig. 1(c), part of this structure has convected out of the field of view and is nearly torn apart. A similar effect is observed for the "blue" eddy in the lower left of Fig. 1(a), which convects upward poloidally (vertically) and is also nearly torn apart by the final frame, Fig. 1(c).

These images of the plasma turbulence reveal details of turbulent structures that are valuable to understanding the fundamental nature of turbulence, yet are not revealed with zero (volume integrated) or one-dimensional (1-D) measurements of density fluctuations. Specific scientific issues that can be uniquely addressed with 2-D images of density fluctuations include comparison of simultaneously-measured flow shearing rates and decorrelation rates, turbulent flow profiles, and nonlinear physics in the edge and core regions of tokamak plasmas. Such nonlinear topics include direct measurement of zonal flows [6], and quantification of nonlinear energy transfer within the turbulent spectrum. Zonal flows, self-generated radically localized potential structures that regulate the turbulence amplitude through time-varying $E \times B$ shear flows, could in principle be observed through time-dependent analysis of the turbulence flow-field [7]. In addition, it has been shown that in order to make quantitative measurements of linear growth rates and nonlinear energy cascades requires 2-D fluctuation data [2].

These images of turbulence in confined tokamak plasmas are helping to provide a more comprehensive picture of the turbulence dynamics than has been previously available. The fluctuation images and their temporal sequence, assembled into movies, demonstrate the dramatic effects of radial shear of the poloidal flow, the strong nonlinear interactions among eddies of different spatial scales, the connecting and shearing of turbulent structures and will be essential to unraveling the complex nonlinear behavior underlying plasma turbulence.

\section{REFERENCES}

[1] B. A. Carreras, "Progress in anomalous transport research in toroidal magnetic confinement devices," IEEE Trans. Plasma Sci., vol. 25, pp. 1281-1321, Dec. 1997.

[2] J. S. Kim, R. D. Durst, R. J. Fonck, E. Fernandez, A. Ware, and P. W. Terry, "Technique for the experimental estimation of nonlinear energy transfer in fully developed turbulence," Phys. Plasmas, vol. 3, no. 11, pp. 3998-4009, 1996.

[3] G. McKee, R. Ashley, R. Durst, R. Fonck, M. Jakubowski, K. Tritz, K. Burrell, C. Greenfield, and J. Robinson, "The beam emission spectroscopy diagnostic on the DIII-D tokamak," Rev. Sci. Instrum., vol. 70, no. 1, pp. 913-916, 1999.

[4] C. Fenzi, R. J. Fonck, M. Jakubowski, and G. R. McKee, "2D turbulence imaging in DIII-D via beam emission spectroscopy," Rev. Sci. Instrum., vol. 72, no. 1, pp. 988-991, 2001.

[5] S. J. Zweben and R. W. Gould, "Structure of edge-plasma turbulence in the Caltech tokamak," Nucl. Fusion, vol. 25, no. 2, pp. 171-183, 1985.

[6] T. S. Hahm, K. H. Burrell, Z. Lin, R. Nazikian, and E. J. Synakowski, "Zonal flow measurements concept I," Plasma Phys. Control Fusion, vol. 42, pp. A205-A210, 2000.

[7] M. Jakubowski, R. J. Fonck, C. Fenzi, and G. R. McKee, "Wavelet-based time-delay estimation for time-resolved turbulent flow analysis," Rev. Sci. Instrum., vol. 72, no. 1, pp. 996-999, 2001. 